\title{
Bioefficacy and Phytotoxicity of Alanto 240 SC (Thiacloprid 240 SC) against Thrips and Natural Enemies in Pomegranate
}

\author{
S.B. Jagginavar, L. Krishna Naik* and S.S. Karabantanal
}

Department of Agricultural Entomology, College of Agriculture, Vijayapur University of Agricultural sciences, Dharwad, Karnataka, India

*Corresponding author

\section{A B S T R A C T}

The experiment on bio efficacy and phytotoxicity of Alanto 240 sc (Thiacloprid 240 SC)

\section{Keywords}

Thrips, Bio efficacy and photo toxicity

\section{Article Info}

Accepted:

10 July 2018

Available Online:

10 August 2018 against thrips and natural enemies in pomegranate was conducted at Krishi Vigyan Kendra farm Vijayapur, Karnataka during 2015-16 by using RBD deigns. During this experiment there was no significant difference among the treatments a day before first spray in recording number of thrips per $10 \mathrm{~cm}$ tender new shoots of pomegranate. At 1 days after first spray significantly lowest number thrips per $10 \mathrm{~cm}$ tender new shoots of pomegranate (2.15) was recorded in higher dose $(1.50 \mathrm{ml} / \mathrm{l})$ of Alanto $240 \mathrm{SC}$ (Thiacloprid 240SC). This higher dose of Alanto $240 \mathrm{SC}(1.25 \mathrm{ml} / \mathrm{l})$ and Cyantraniliprole $10.26 \%$ OD @ 0.75ml/1 treatments were on par with each other in reducing the thrips populations. At 3 days after the first spray the higher dose of Alanto $240 \mathrm{SC}(1.25 \mathrm{ml} / \mathrm{l})$ and Cyantraniliprole $10.26 \%$ OD @ $0.75 \mathrm{ml} / 1$ recorded 2.00 and 2.13 thrips population per $10 \mathrm{~cm}$ tender new shoots of pomegranate, respectively and they were statistically at par with each other. Seven, ten and fifteen days after spray were also recorded similar trend in recording thrips populations.

\section{Introduction}

Pomegranate (Punica granatum) is grown in tropical and subtropical regions of the world. The total area under cultivation of pomegranate in India is 107.00 thousand ha and production is around 743.00 thousand tons. Maharashtra is the leading producer of pomegranate followed by Karnataka, Andhra Pradesh, Gujarat and Tamil Nadu. Ganesh, Bhagwa, Ruby, Arakta and Mridula are the different varieties of pomegranates produced in Maharashtra. In India, pomegranate is commercially cultivated in Solapur, Sangli, Nasik, Ahmednagar, Pune, Dhule,
Aurangabad, Satara, Osmanabad and Latur districts of Maharashtra; Bijapur, Belgaum and Bagalkot districts of Karnataka, Zirpe, 1966 and Mote et al., 1992). Most problematic and economically important insect pests in the production of appropriate quality fruits of pomegranate for domestic and export markets are fruit borer (pomegranate butterfly) and thrip Scirtothrips dorsalis Hood. Halleppanvar (1955) reported 100 per cent damage to pomegranate under severe epidemic conditions in Karnataka. The species of thrip (Scirtothrips dorsalis Hood.) infesting pomegranate is a polyphagous pest known to cause damage to several seasonal field crops, 
vegetables and fruit crops. Both nymph and adult of thrip feed by rasping and sucking type of mouth parts. They lacerate the surface of developing fruiting parts and cause deformation showing corcky appearance on surface of fruits which ultimately deteriorate the quality, fetching low price in domestic market and not accepted for export.

Hence to minimize the thrips population present study have been undertaken to evaluate the bioefficacy, phytotoxocity and of different newer insecticides at different doses against thrips and natural enemies in pomegranate orchard.

\section{Materials and Methods}

The experiment on pomegranate variety Kesar was conducted at College of Agriculture, Vijayapur, Karnataka during 2015-16 by using RBD deign in 4 replication by adopting the plot size $18 \mathrm{X} 18 \mathrm{mt}$ in irrigated red soil.

\section{Thrips population}

Total Number of thrips per $10 \mathrm{~cm}$ tender new shoots was observed on ten randomly selected leaves per plot at one day before and 3, 7, 10 $\& 15$ days after each spray.

\section{Natural enemies}

Recorded natural enemies such as Coccinellids / plant on 5 randomly selected plants at one day before and 3, 7, $10 \& 15$ days after each spray and average values were worked out.

\section{Phytotoxicity}

Phytotoxic effects of alanto 240 SC (thiacloprid 240SC) at 1.50 and $3.00 \mathrm{ml} / 1$. in comparison with untreated control were recorded on 0 to 10 scale at $1,3,5,7$ and 10 days after spray.

\section{Yield}

Marketable yield after harvest at different pickings per pomegranate tree was converted to tones per hectare. Data were analyzed statistically

\section{Results and Discussion}

\section{Bio-efficacy of Alanto 240 SC (Thiacloprid 240SC) against thrips on pomegranate}

The data presented in the table 1 indicated that there was no significant difference among the treatments a day before first spray in recording number of thrips per $10 \mathrm{~cm}$ tender new shoots of pomegranate.

At 1 days after first spray significantly lowest number thrips per $10 \mathrm{~cm}$ tender new shoots of pomegranate (2.15) was recorded in higher dose $(1.50 \mathrm{ml} / \mathrm{l})$ of Alanto $240 \mathrm{SC}$ (Thiacloprid 240SC). This higher dose of Alanto 240 SC $(1.50 \mathrm{ml} / \mathrm{l})$ and Cyantraniliprole $10.26 \%$ OD ( $0.75 \mathrm{ml} / 1$ treatments were on par with each other in reducing the thrips populations this finding is at par with the findings of Din N, Ashraf M, Hussain S (2016) against thrips in brinjal. At 3 days after the first spray the higher dose of Alanto $240 \mathrm{SC}(1.50 \mathrm{ml} / \mathrm{l})$ and Cyantraniliprole $10.26 \%$ OD @ $0.75 \mathrm{ml} / \mathrm{l}$ recorded 2.00 and 2.13 thrips population per $10 \mathrm{~cm}$ tender new shoots of pomegranate, respectively and they were statistically at par with each other. Seven and ten days after spray also recorded similar trend in recording thrips populations. At 15 days after spray, the thrips populations were increased in all treatments (Table 2). Similar trend was noticed in second spray also where in higher dose of Alanto $240 \mathrm{SC}(1.50 \mathrm{ml} / \mathrm{l})$ and Cyantraniliprole $10.26 \%$ OD @ $0.75 \mathrm{ml} / 1$ superior in reducing pomegranate thrips population in pomegranate orchard is at par with findings of Ghelani and et al., (2014) (Table 3). 
Table.1 Treatment details

\begin{tabular}{|l|l|c|}
\hline SI. No & Treatments & Dose (ml/liter) \\
\hline 1 & Alanto 240 SC (Thiacloprid 240SC) & 1.00 \\
\hline 2 & Alanto 240 SC (Thiacloprid 240SC) & 1.25 \\
\hline 3 & Alanto 240 SC (Thiacloprid 240SC) & 1.50 \\
\hline 4 & Cyantraniliprole 10.26 \% OD & 0.75 \\
\hline 6 & Imdacloprid 17.8\%SL & 0.25 \\
\hline 7 & Untreated control & - \\
\hline
\end{tabular}

Table.2 Bio-efficacy of Alanto 240 SC (Thiacloprid 240SC) against pomegranate thrips (First spray)

\begin{tabular}{|c|c|c|c|c|c|c|c|c|}
\hline \multirow{2}{*}{$\begin{array}{l}\text { SI. } \\
\text { No }\end{array}$} & \multirow[t]{2}{*}{ Treatments } & \multirow{2}{*}{$\begin{array}{c}\text { Dose } \\
(\mathrm{ml} / \mathrm{l})\end{array}$} & \multicolumn{6}{|c|}{ Number of thrips per $10 \mathrm{~cm}$ tender new shoots } \\
\hline & & & $1 \mathrm{DBS}$ & 1DAS & 3DAS & 7 DAS & 10 DAS & 15DAS \\
\hline 1. & $\begin{array}{l}\text { Alanto } 240 \text { SC (Thiacloprid } \\
\text { 240SC) }\end{array}$ & 1.00 & $\begin{array}{l}11.75 \\
(3.57)\end{array}$ & $\begin{array}{l}4.93 \\
(2.44)\end{array}$ & $\begin{array}{c}4.45 \\
(2.33)\end{array}$ & $\begin{array}{l}4.33 \\
(2.31)\end{array}$ & $\begin{array}{c}4.13 \\
(2.26)\end{array}$ & $3.97(2.33)$ \\
\hline 2. & $\begin{array}{l}\text { Alanto } 240 \text { SC (Thiacloprid } \\
\text { 240SC) }\end{array}$ & 1.25 & $\begin{array}{l}12.50 \\
(3.67)\end{array}$ & $\begin{array}{c}3.98 \\
(2.23)\end{array}$ & $\begin{array}{c}3.64 \\
(2.15)\end{array}$ & $\begin{array}{c}3.31 \\
(2.08)\end{array}$ & $\begin{array}{l}3.20 \\
(2.05)\end{array}$ & $3.38(2.09)$ \\
\hline 3. & $\begin{array}{l}\text { Alanto } 240 \text { SC (Thiacloprid } \\
\text { 240SC) }\end{array}$ & 1.50 & $\begin{array}{l}11.25 \\
(3.50)\end{array}$ & $\begin{array}{c}2.15 \\
(1.77)\end{array}$ & $\begin{array}{c}2.00 \\
(1.73)\end{array}$ & $\begin{array}{c}1.87 \\
(1.69)\end{array}$ & $\begin{array}{l}1.85 \\
(1.69)\end{array}$ & $1.87(1.69)$ \\
\hline 4. & $\begin{array}{l}\text { Cyantraniliprole } 10.26 \% \\
\text { OD }\end{array}$ & 0.75 & $\begin{array}{l}10.75 \\
(3.43)\end{array}$ & $\begin{array}{l}2.25 \\
(1.80)\end{array}$ & $\begin{array}{c}2.13 \\
(1.77)\end{array}$ & $\begin{array}{c}2.13 \\
(1.77)\end{array}$ & $\begin{array}{c}2.68 \\
(1.92)\end{array}$ & $2.23(1.80)$ \\
\hline 5. & Imdacloprid $17.8 \% \mathrm{SL}$ & 0.25 & $\begin{array}{l}13.75 \\
(4.69)\end{array}$ & $\begin{array}{l}3.03 \\
(2.74)\end{array}$ & $\begin{array}{l}2.95 \\
(2.71)\end{array}$ & $\begin{array}{c}2.80 \\
(2.66)\end{array}$ & $\begin{array}{l}2.75 \\
(2.65)\end{array}$ & $\begin{array}{c}2.78 \\
(2.63)\end{array}$ \\
\hline 6. & Untreated control & - & $\begin{array}{l}12.25 \\
(3.64)\end{array}$ & $\begin{array}{l}11.50 \\
(3.54)\end{array}$ & $\begin{array}{l}11.71 \\
(3.57)\end{array}$ & $\begin{array}{l}11.75 \\
(3.57)\end{array}$ & $\begin{array}{l}11.99 \\
(3.60)\end{array}$ & $\begin{array}{l}11.88 \\
(3.59)\end{array}$ \\
\hline 7. & $\mathrm{SEm} \pm$ & & 0.15 & 0.11 & 0.11 & 0.13 & 0.12 & 0.13 \\
\hline 8. & $\mathrm{CD}$ at $5 \%$ & & NS & 0.33 & 0.33 & 0.40 & 0.37 & 0.41 \\
\hline 9. & $\mathrm{CV} \%$ & & 6.55 & 7.15 & 7.32 & 8.80 & 8.27 & 9.14 \\
\hline
\end{tabular}

DBS $=$ Days before spray DAS $=$ Days after spray, Values in the brackets are $\sqrt{ }(x+1)$

Table.3 Bio-efficacy of Alanto 240 SC (Thiacloprid 240 SC) against pomegranate thrips (Second pray)

\begin{tabular}{|c|c|c|c|c|c|c|c|c|}
\hline \multirow{2}{*}{$\begin{array}{l}\text { Sl. } \\
\text { No }\end{array}$} & \multirow[t]{2}{*}{ Treatments } & \multirow{2}{*}{$\begin{array}{l}\text { Dose } \\
(\mathrm{ml} / \mathrm{l})\end{array}$} & \multicolumn{6}{|c|}{ Number of thrips per $10 \mathrm{~cm}$ tender new shoots } \\
\hline & & & 1 DBS & 1DAS & 3DAS & 7 DAS & 10 DAS & 15DAS \\
\hline 1. & $\begin{array}{l}\text { Alanto } 240 \text { SC (Thiacloprid } \\
\text { 240SC) }\end{array}$ & 1.00 & $\begin{array}{l}11.25 \\
(3.50)\end{array}$ & $\begin{array}{l}4.93 \\
(2.44)\end{array}$ & $\begin{array}{l}3.93 \\
(2.22)\end{array}$ & $\begin{array}{l}3.80 \\
(2.19)\end{array}$ & $\begin{array}{l}3.75 \\
(2.18)\end{array}$ & $\begin{array}{l}3.95 \\
(2.22)\end{array}$ \\
\hline 2. & $\begin{array}{l}\text { Alanto } 240 \text { SC (Thiacloprid } \\
\text { 0SC) }\end{array}$ & 1.25 & $\begin{array}{l}12.50 \\
(3.67)\end{array}$ & $\begin{array}{l}3.98 \\
(2.23)\end{array}$ & $\begin{array}{l}3.50 \\
(2.12)\end{array}$ & $\begin{array}{l}3.60 \\
(2.14)\end{array}$ & $\begin{array}{l}3.13 \\
(2.03)\end{array}$ & $\begin{array}{l}3.02 \\
(2.00)\end{array}$ \\
\hline 3. & $\begin{array}{l}\text { Alanto } 240 \text { SC (Thiacloprid } \\
\text { 240SC) }\end{array}$ & 1.50 & $\begin{array}{l}11.00 \\
(3.46)\end{array}$ & $\begin{array}{l}2.15 \\
(1.77)\end{array}$ & $\begin{array}{l}1.75 \\
(1.66)\end{array}$ & $\begin{array}{l}1.68 \\
(1.64)\end{array}$ & $\begin{array}{l}1.58 \\
(1.61)\end{array}$ & $\begin{array}{l}1.61 \\
(1.62)\end{array}$ \\
\hline 4. & Cyantraniliprole $10.26 \%$ OD & 0.75 & $\begin{array}{l}10.75 \\
(3.43)\end{array}$ & $\begin{array}{l}2.75 \\
(1.94)\end{array}$ & $\begin{array}{l}2.02 \\
(1.74)\end{array}$ & $\begin{array}{l}1.95 \\
(1.72)\end{array}$ & $\begin{array}{l}1.83 \\
(1.68)\end{array}$ & $\begin{array}{l}1.93 \\
(1.71)\end{array}$ \\
\hline 5. & Imdacloprid $17.8 \% \mathrm{SL}$ & 0.25 & $\begin{array}{l}14.75 \\
(4.83)\end{array}$ & $\begin{array}{l}3.03 \\
(2.73)\end{array}$ & $\begin{array}{l}2.38 \\
(2.51)\end{array}$ & $\begin{array}{l}2.28 \\
(2.48)\end{array}$ & $\begin{array}{l}2.15 \\
(2.44)\end{array}$ & $\begin{array}{l}2.08 \\
(2.42)\end{array}$ \\
\hline 6. & Untreated control & - & $\begin{array}{l}12.25 \\
(3.64)\end{array}$ & $\begin{array}{l}11.50 \\
(3.54)\end{array}$ & $\begin{array}{l}11.75 \\
(3.57)\end{array}$ & $\begin{array}{l}11.78 \\
(3.57)\end{array}$ & $\begin{array}{l}11.90 \\
(3.59)\end{array}$ & $\begin{array}{l}12.03 \\
(3.61)\end{array}$ \\
\hline 7. & $\mathrm{SEm} \pm$ & & 0.17 & 0.18 & 0.14 & 0.14 & 0.12 & 0.14 \\
\hline 8. & $\mathrm{CD}$ at $5 \%$ & & NS & 0.53 & 0.41 & 0.43 & 0.43 & 0.41 \\
\hline 9. & $\mathrm{CV} \%$ & & 7.45 & 11.54 & 9.46 & 9.74 & 8.60 & 9.57 \\
\hline
\end{tabular}

DBS $=$ Days before spray DAS $=$ Days after spray, Values in the brackets are $\sqrt{ }(x+1)$ 
Table.4 Bio-efficacy of Alanto 240 SC (Thiacloprid 240SC) against pomegranate thrips and pomegranate fruit yield

\begin{tabular}{|c|l|c|c|}
\hline SI. No & \multicolumn{1}{|c|}{ Treatments } & Dose $(\mathbf{m l} / \mathbf{l})$ & Fruits yield (tonnes/ha) \\
\hline $\mathbf{1}$ & Alanto 240 SC (Thiacloprid 240SC) & 1.00 & $\mathbf{8 . 7 5}$ \\
\hline $\mathbf{2}$ & Alanto 240 SC (Thiacloprid 240SC) & 1.25 & 9.69 \\
\hline $\mathbf{3}$ & Alanto 240 SC (Thiacloprid 240SC) & 1.50 & $\mathbf{1 2 . 0 0}$ \\
\hline $\mathbf{4}$ & Cyantraniliprole 10.26 \% OD & 0.75 & $\mathbf{1 0 . 5 0}$ \\
\hline $\mathbf{5}$ & Imdacloprid 17.8\%SL & 0.25 & $\mathbf{1 0 . 2 5}$ \\
\hline $\mathbf{6}$ & Untreated control & - & $\mathbf{8 . 2 5}$ \\
\hline 7 & S.Em \pm & & $\mathbf{0 . 4 5}$ \\
\hline $\mathbf{8}$ & CD at 5\% & & $\mathbf{1 . 3 7}$ \\
\hline $\mathbf{9}$ & CV \% & $\mathbf{9 . 1 5}$ \\
\hline
\end{tabular}

DBS $=$ Days before spray DAS $=$ Days after spray

Table.5 Bio-efficacy of Alanto 240 SC (Thiacloprid 240SC) on natural enemies in pomegranate ecosystem

\begin{tabular}{|c|c|c|c|c|c|c|c|}
\hline \multirow{2}{*}{$\begin{array}{l}\text { SI. } \\
\text { No }\end{array}$} & \multirow[t]{2}{*}{ Treatments } & \multirow{2}{*}{$\begin{array}{l}\text { Dosage } \\
\text { (ml/ha) }\end{array}$} & \multicolumn{5}{|c|}{ No. of Coccinellids sps/plant } \\
\hline & & & 1 DBS & 3 DAS & 7 DAS & 10DAS & 15DAS \\
\hline 1 & Alanto 240 SC (Thiacloprid 240SC) & 1.0 & 5.75 & 5.25 & 5.00 & 5.03 & 5.28 \\
\hline 2 & Alanto 240 SC (Thiacloprid 240SC) & 1.25 & 5.00 & 4.75 & 4.50 & 4.75 & 4.88 \\
\hline 3 & Alanto 240 SC (Thiacloprid 240SC) & 1.50 & 5.50 & 5.19 & 4.94 & 4.69 & 4.81 \\
\hline 4 & Cyantraniliprole $10.26 \%$ OD & 0.75 & 5.50 & 5.13 & 5.13 & 5.25 & 5.38 \\
\hline 5 & Imdacloprid $17.8 \% \mathrm{SL}$ & 0.25 & 5.25 & 4.75 & 4.75 & 4.88 & 5.00 \\
\hline 6 & Untreated control & - & 5.50 & 5.75 & 5.88 & 6.00 & 6.19 \\
\hline 7 & $\mathrm{~S} . \mathrm{Em} \pm$ & - & 0.40 & 0.42 & 0.40 & 0.34 & 0.38 \\
\hline 8 & $\mathrm{CD}$ at $5 \%$ & - & NS & NS & NS & NS & NS \\
\hline 9 & CV\% & - & 14.82 & 16.23 & 15.80 & 13.25 & 14.63 \\
\hline
\end{tabular}

Note: DBS = Day before spray

DAS = Days after spray C: Coccinellids sps

Table.6 Phytotoxicity of Alanto 240 SC (Thiacloprid 240SC) on pomegranate plants

\begin{tabular}{|c|c|c|c|c|c|c|c|c|c|}
\hline \multirow{2}{*}{$\begin{array}{l}\text { Sl. } \\
\text { No }\end{array}$} & \multirow[t]{2}{*}{ Treatments } & \multirow{2}{*}{$\begin{array}{c}\text { Dosage } \\
(\mathrm{ml} / \mathrm{l})\end{array}$} & \multicolumn{7}{|c|}{ Phytotoxicity symptoms * } \\
\hline & & & $\begin{array}{c}\text { Leaf } \\
\text { chlorosis }\end{array}$ & $\begin{array}{l}\text { Leaf tip } \\
\text { burning }\end{array}$ & $\begin{array}{c}\text { Leaf } \\
\text { necrosis }\end{array}$ & $\begin{array}{c}\text { Leaf } \\
\text { epinasty }\end{array}$ & $\begin{array}{c}\text { Leaf } \\
\text { hyponasty }\end{array}$ & $\begin{array}{l}\text { Vein } \\
\text { clearing }\end{array}$ & $\begin{array}{l}\text { Wilting \& } \\
\text { Resetting }\end{array}$ \\
\hline 1. & $\begin{array}{l}\text { Alanto } 240 \text { SC } \\
\text { (Thiacloprid 240SC) }\end{array}$ & 1.50 & 0.0 & 0.0 & 0.0 & 0.0 & 0.0 & 0.0 & 0.0 \\
\hline 2. & $\begin{array}{l}\text { Alanto } 240 \text { SC } \\
\text { (Thiacloprid 240SC) }\end{array}$ & 3.00 & 0.0 & 0.0 & 0.0 & 0.0 & 0.0 & 0.0 & 0.0 \\
\hline 3. & Untreated control & - & 0.0 & 0.0 & 0.0 & 0.0 & 0.0 & 0.0 & 0.0 \\
\hline
\end{tabular}

* Phytotoxicity scale of 0 to 10 was followed.

* Phytotoxicity was observed at $1,3,7,10$ and 15 days after spray. 


\section{Pomegranate fruit yield}

Higher dose of Alanto 240 SC $(1.25 \mathrm{ml} / \mathrm{l})$ recorded 12.00 tonnes per hectare yield which was on par with Cyantraniliprole $10.26 \%$ OD @ 0.75ml/1 (10.50) (Table 4).

\section{Bio-efficacy of Alanto 240 SC (Thiacloprid 240SC) on natural enemies in pomegranate ecosystem}

The test molecule Alanto 240 SC (Thiacloprid 240SC) @ 1.00, 1.25 and $1.50 \mathrm{ml} / 1$ was evaluated against the natural enemies like coccinellids in comparison with Cyantraniliprole $10.26 \%$ OD @ 0.75ml/1 and untreated control (water spray). Results indicated that there was no significant difference among the treatments at three, seven, ten and fifteen days after spray (Table $5)$.

Phytotoxicity of Alanto 240 SC (Thiacloprid 240SC) on pomegranate plants

Application of Alanto 240 SC (Thiacloprid 240SC)@ $1.50 \mathrm{ml} / \mathrm{L}$ (0.36 g a.i/lit) and 3.00 $\mathrm{ml} / \mathrm{L}(0.72 \mathrm{~g}$ a.i/lit) did not result in any phytotoxicity symptoms such as leaf chlorosis, leaf tip burning, leaf necrosis, leaf epinasty, leaf hyponasty vein clearing, wilting and rosetting recorded at 1, 3, 5,7 and 10 days after application were not observed (Table 5).

Among different treatments, Alanto 240 SC (Thiacloprid 240SC)@1.50 ml/L was found effective in reducing the thrips population with higher yield in pomegranate. Further, there was no phytotoxic effect of Alanto 240 SC (Thiacloprid 240SC)@3.00 ml/L (0.72 g a.i/lit) pomegranate on plants.

\section{References}

Din N, Ashraf M, Hussain S. Effect of different nonchemical and chemical measures against onion thrips. Journal of Entomology and Zoology Studies. 2016; 4(5): 10-12

Ghelani MK, Kabaria BB, Chhodavadia SK. Field efficacy of various insecticides against major sucking pests of $\mathrm{Bt}$ cotton. Journal of Biopesticides. 2014; 5(1): 1-3

Halleppanvar, N. L. 1956. Important insect pests of fruit crops in Bombay, Karnataka and their control. Farmer Bombay, 7 (6): 95.

Zirpe, A. G. 1966. Pests of pomegranate and their control in Maharashtra State, M. Sc. (Agri.), Thesis, University of Poona, Maharashtra

\section{How to cite this article:}

Jagginavar, S.B., L. Krishna Naik and Karabantanal, S.S. 2018. Bioefficacy and Phytotoxicity of Alanto 240 SC (Thiacloprid 240 SC) against Thrips and Natural Enemies in Pomegranate. Int.J.Curr.Microbiol.App.Sci. 7(08): 1598-1602. doi: https://doi.org/10.20546/ijcmas.2018.708.182 